\title{
Politiques de pêche et innovations adaptatives des pêcheries artisanales sénégalaises
}

\author{
Moustapha Dème* et Djiga Thiao (D) \\ Économie des pêches, économie de l'environnement, Centre de recherches océanographiques de Dakar-Thiaroye (CRODT/ \\ ISRA), Dakar, Sénégal
}

Reçu le 3 septembre 2018. Accepté le 30 juillet 2020

Les enjeux de soutenabilité doivent trouver certaines de leurs réponses dans les innovations techniques. C'est l'arrière-plan de l'analyse proposée par les auteurs des processus d'innovation dans la pêche artisanale sénégalaise, un secteur crucial pour le pays en termes d'emplois, de revenus et de fourniture de protéines. Après avoir dressé un bilan mitigé des politiques de modernisation menées par les pouvoirs publics depuis les années 1960, les auteurs passent en revue les innovations techniques introduites régulièrement dans leurs activités par les pêcheurs artisans pour faire face aux baisses de rendement et au renchérissement des coûts de production qu'ils subissent. Si la soutenabilité économique et sociale de la pêche artisanale a ainsi été assurée, la pression sur les ressources halieutiques s'en est trouvée grandement accrue. La soutenabilité écologique de la pêche artisanale sénégalaise reste posée.

La Rédaction

Résumé - Ces dernières décennies, les pêcheries artisanales sénégalaises ont été marquées par d'importantes innovations en réponse aux politiques de pêche et aux fluctuations de l'abondance des ressources. Cet article aborde l'adéquation des politiques avec les contraintes et les enjeux du secteur dans une perspective d'intégration des innovations dans la prise de décision. Il apparaît que les tentatives de modernisation en remplaçant les pirogues par des bateaux semi-industriels ont échoué. La capacité des pêcheurs à considérer de nouvelles innovations et stratégies a pu garantir plus de viabilité et de résilience à leurs activités. L'adaptabilité leur a permis de réagir à divers chocs exogènes d'ordre économique et à la variabilité de l'abondance et de la demande en poisson. Ces interactions adaptatives entre capital naturel et capital humain confortent la notion de «durabilité faible» dans le domaine de la pêche.

Mots-clés : pêche artisanale / Sénégal / innovations adaptatives / durabilité

\begin{abstract}
Fisheries policies and adaptive innovations in the Senegalese artisanal fisheries. Over recent decades, the Senegalese artisanal fisheries have undergone major innovations in response to fishing policies and fluctuations in fish abundance. This article addresses the adequacy of public policies regarding the constraints and challenges in the fisheries sector with a view to integrating innovations into decision-making processes. Since these changes are essentially due to economic factors, we addressed the issue mainly from an economic angle. Our results demonstrate that attempts to modernize the artisanal fisheries by replacing for example the traditional canoes by semi-industrial boats have failed. In fact, the ability of artisanal fishers to adopt new innovations and more effective strategies was able to guarantee a greater viability and resilience of their activities. More generally, their adaptability allowed them to suitably react to various exogenous shocks related to the economic aspects as well as to the variability of fish abundance and demand. These adaptive interactions between natural capital and human capital which govern the development of the artisanal fisheries support the notion of 'weak sustainability' in the field of marine fisheries.
\end{abstract}

Keywords: artisanal fisheries / Senegal / adaptive innovations / sustainability

\footnotetext{
*Auteur correspondant : moustapha.deme@gmail.com
} 
Même s'il a été d'abord principalement perçu sous l'angle technologique, le concept d'innovation est large et multiforme et couvre des aspects non technologiques ${ }^{1}$. Dans le Manuel d'Oslo consacré à ce concept, l'OCDE (2005) définit l'innovation comme la mise en œuvre d'un produit ou d'un procédé nouveau ou sensiblement amélioré, d'une nouvelle méthode de commercialisation ou d'une nouvelle méthode organisationnelle dans les pratiques de l'entreprise, l'organisation du lieu de travail ou les relations extérieures. Quatre catégories d'innovation sont donc considérées, à savoir l'innovation de produit (bien ou service), de procédé (production ou distribution), d'organisation (introduction de nouvelles méthodes) et de commercialisation (changement de stratégie). Dans l'évolution de l'histoire humaine, les innovations radicales ont façonné les grandes mutations du monde et les innovations progressives alimentent de manière continue le changement économique. Même dans le domaine de la pêche, ces quatre types d'innovation ont permis de développer et de perpétuer ce secteur malgré les différentes contraintes. L'innovation est même considérée comme un impératif pour la sécurité alimentaire dans les pays en développement (Hall et al., 2013) et une opportunité pour améliorer la gestion des pêches (Bradley et al., 2019).

Au Sénégal, le secteur de la pêche se caractérise depuis quelques années par un contexte de forte pression entraînant la surexploitation des principaux stocks halieutiques. Cette situation s'est traduite par l'éloignement des zones de pêche, le renchérissement des coûts de production et la baisse des rendements. C'est dans ce contexte que sont nées les importantes mutations intervenues dans les pêcheries artisanales sénégalaises au cours de ces dernières décennies. Elles sont généralement le fruit des réponses des pêcheurs artisans, d'une part, aux politiques instituées par les autorités publiques en charge de la pêche et, d'autre part, aux fluctuations à moyen et à long terme de l'abondance des ressources halieutiques. Le dynamisme de la pêche artisanale combiné aux différentes politiques publiques de soutien au secteur de la pêche se traduit aujourd'hui par une surexploitation de la plupart des stocks de poisson. Sur les ressources démersales côtières et les

\footnotetext{
${ }^{1}$ La recherche qui a fait l' 'objet de cet article trouve son origine dans la réflexion stratégique que le Centre de recherches océanographiques de Dakar-Thiaroye (CRODT) mène régulièrement dans ses programmes de recherches afin de fournir les bases scientifiques de la gestion durable des ressources halieutiques. Ainsi, au cours de ces dernières années, pour mieux appuyer les décideurs et les professionnels de la pêche, le CRODT s'est donné comme priorité majeure de conduire une analyse diachronique et paradigmatique sur les innovations adaptatives dans les pêcheries artisanes sénégalaises dans le but d'accompagner les nouveaux chantiers de réforme de la politique de pêche au Sénégal.
}

petits pélagiques côtiers en particulier, des signes de surexploitation ont été notés aussi bien de façon scientifique (évaluations des stocks par le CRODT et le COPACE ${ }^{2}$ ) qu'empirique (observations sur le terrain). Cette situation menace la durabilité de l'exploitation halieutique au Sénégal.

Ce qui a été tout à fait remarquable dans les péripéties des pêcheries artisanales, c'est que leur capacité intrinsèque d'adaptation a toujours été le principal socle de leur développement et a permis d'assurer leur résilience face aux différentes crises. D'ailleurs, les politiques publiques en matière de pêche artisanale n'ont pas fondamentalement évolué ces trente dernières années et ont concerné surtout les actions de modernisation (construction d'infrastructures de débarquements conformes aux normes des pays importateurs), la détaxe des équipements de pêche (filets de pêche, moteur hors-bord) et la subvention sur le carburant pêche. La plupart des initiatives formelles de modernisation pilotées par l'État et auxquelles les pêcheurs artisans n'ont pas adhéré ont généralement eu des résultats mitigés voire infructueux malgré les importants moyens mobilisés. À l'opposé, les différentes innovations impulsées par les communautés de pêcheurs ont pu générer des dynamiques adaptatives qui ont été le gage de l'efficacité des pêcheries artisanales. Elles ont également permis d'atténuer les effets négatifs de la raréfaction des ressources halieutiques durant ces dernières décennies. Il nous paraît donc judicieux de documenter le contexte historique et la nature de ces innovations adaptatives et de caractériser leurs conséquences par rapport aux modèles de durabilité classiquement définis dans la littérature.

Dans cet article, après avoir présenté la méthodologie de notre étude, nous passerons en revue les programmes majeurs mis en œuvre par l'État pour moderniser les pêcheries artisanales. Ensuite nous procéderons à une analyse détaillée des principales innovations fonctionnelles et opérationnelles qui ont marqué les pêcheries artisanales sénégalaises. Enfin, nous mettrons un accent particulier sur les conséquences de ces innovations, notamment en termes d'efficacité, d'adaptabilité et surtout de durabilité. Tenant compte des contraintes et des enjeux actuels du secteur de la pêche, notre réflexion nous a conduits à proposer des stratégies permettant de mieux intégrer les innovations adaptatives dans les processus de prise de décisions et de mise en œuvre des politiques de gestion des ressources halieutiques au Sénégal.

${ }^{2}$ CRODT: Centre de recherches océanographiques de DakarThiaroye; COPACE: Comité des pêches pour l'Atlantique Centre-Est. 


\section{Matériel et méthode}

\section{Revue documentaire}

En se basant sur la littérature, cette étude fait le point sur les politiques publiques phares de gestion et de développement des pêcheries artisanales en rapport avec leurs objectifs visés. Ainsi, pour chacune d'entre elles, nous avons évalué les écarts entre les objectifs définis et les impacts générés. Les facteurs qui ont favorisé les effets imprévus et les goulots d'étranglement ont aussi été aussi identifiés et analysés. En outre, pour mieux cerner la portée des politiques de pêche et les mutations opérées, la dynamique d'exploitation des ressources a été appréhendée sous l'angle de la dimension économique. En effet, les facteurs économiques constituent le principal socle de la plupart des mutations notées dans la pêche artisanale. Par ailleurs, en s'inspirant également de la littérature (Solow, 1993 ; Faucheux et Noël, 1995 ; Godard, 1994), nous avons pu mieux cerner la grande adaptabilité de la pêche artisanale par rapport à la notion de «durabilité faible» dans l'exploitation des ressources halieutiques.

\section{Entretiens avec des pêcheurs}

Notre démarche s'est fondée, d'une part, sur la mise à jour des connaissances et, d'autre part, sur l'analyse chronologique des politiques de pêche et des mutations des systèmes d'exploitation. Ainsi, en plus de la réalisation d'une revue documentaire, nous nous sommes entretenus avec différents acteurs de la pêche artisanale afin de mieux cerner leur évolution, par exemple, en 2018, avec quelques personnes choisies de manière raisonnée (key informant interviews) du fait de leurs expériences pratiques et leurs connaissances de l'histoire de l'activité. Il s'agissait notamment de vieux pêcheurs restés actifs dans le secteur en tant que propriétaires ou gestionnaires d'unités de production. Ainsi, nous avons pu discuter avec 30 personnes à Saint Louis, Kayar, Yoff, Hann, Mbour et Joal. Elles nous ont fait part de leurs observations, de leurs appréciations sur les différentes politiques et leurs impacts réels.

\section{Observations in situ et exploitation de données halieutiques}

Des observations sur le terrain ont également permis de comprendre la réalité actuelle de l'exploitation artisanale des ressources halieutiques sénégalaises. Pour mieux étayer l'analyse des mutations, des sources de données ont aussi été explorées. Il s'agit notamment des bases de données de la Direction des pêches maritimes (DPM) et du Centre de recherches océanographiques de Dakar-Thiaroye (CRODT). Les données mises à contribution à ce niveau concernent surtout les caractéristiques de la flottille de pêche et le volume des débarquements de 1981 à 2015.

\section{Les programmes de modernisation de la pêche artisanale}

\section{Les hypothèses de base de la modernisation de la pêche artisanale}

Au lendemain de l'indépendance du Sénégal en 1960 et jusqu'au début des années 1980, la volonté des autorités publiques était de développer quasi exclusivement la pêche industrielle. Dans les quatre premiers plans quinquennaux de développement économique et social du Sénégal (1960-1977), au moins $82 \%$ des fonds affectés au secteur de la pêche (23,8 milliards de FCFA) ont été alloués à la pêche industrielle (Dème, 1991b). L'intervention publique était essentiellement centrée sur le segment «capture». Ainsi la Société sénégalaise d'armement à la pêche (SOSAP), dotée d'une flottille de 28 unités (18 senneurs ciblant les petits pélagiques et 10 canneurs ciblant les thons) sur la période 1965-1975 (Domingo, 1982; Charneau, 1988), a-t-elle bénéficié de l'essentiel de l'enveloppe financière prévue pour la pêche industrielle. Les faibles montants alloués à la pêche artisanale ont été engagés dans une politique de modernisation de ce sous-secteur jugé archaïque et peu productif.

La vision des pouvoirs politiques de l'époque était que la pêche artisanale devait évoluer vers des formes industrielles plus performantes (Chauveau, 1989). Ainsi, dans les trois premiers plans (1960-1973), les fonds prévus pour la pêche artisanale (1,9 milliard de FCFA, soit $14,8 \%$ du budget total pêche) ont été affectés à l'acquisition de sardiniers et cordiers dans la perspective d'une modernisation radicale. Les sardiniers sont des bateaux de pêche spécialisés dans la capture des petits pélagiques comme la sardine et les sardinelles, des poissons vivant près de la surface des eaux. Les cordiers, eux, sont utilisés pour une technique de pêche basée sur un dispositif constitué d'un ensemble de cordages très résistants de 6 à $8 \mathrm{~mm}$ de diamètre assemblés les uns aux autres auxquels sont amarrés des avançons munis $\mathrm{d}$ 'hameçons et ceci tous les $3,50 \mathrm{~m}$ environ.

Comparés aux pirogues sénégalaises de l'époque, ces prototypes d'embarcation de type semi-industriel sont d'une capacité et d'une autonomie plus grande, ils sont aussi dotés de systèmes de détection du poisson plus ou moins sophistiqués. Les sardiniers devaient exploiter les stocks de sardinelles côtiers migrateurs et tout autre stock de petits pélagiques peu accessible à la pêche artisanale (Dème et Levenez, 1991). Les cordiers, par contre, 
devaient permettre l'élargissement et la diversification de la pêche piroguière en capturant des espèces à fortes valeurs commerciales peu ou pas pêchées par les pirogues (Kébé, 1982). Ainsi, à terme, sardiniers et cordiers devaient supplanter respectivement les unités artisanales exploitant les petits pélagiques côtiers et celles ciblant les espèces démersales côtières.

\section{Le programme cordier}

Le programme cordier a débuté en 1963 et s'est étalé quasiment sur deux décennies. Les objectifs qui lui ont été assignés ont évolué dans le temps. Initialement, il s'agissait de mettre à la disposition des pêcheurs artisans des moyens de production plus modernes leur permettant d'augmenter leurs captures en accédant à de nouvelles zones de pêche. Le besoin de décentraliser la flottille de cordiers vers les ports secondaires existants (SaintLouis) ou à construire (Kafountine, Elinkine, Djifère) est ensuite avancé. Les formes organisationnelles de gestion et d'administration du programme ont connu plusieurs mutations. Si la Direction de l'océanographie et des pêches maritimes (DOPM) a initié le programme, pour les besoins d'une pérennisation, elle a ensuite rétrocédé la gestion des bateaux cordiers aux pêcheurs et armateurs regroupés au sein de différentes coopératives et groupements d'intérêt économique (GIE).

Si les premiers bateaux cordiers sont en bois, les prototypes qui ont suivi sont, eux, en métal. La flottille de cordiers est constituée de petits bateaux mesurant $13 \mathrm{~m}$ de longueur et 3,5 à 4,5 m de largeur. Dotées d'un moteur de 75 à $160 \mathrm{cv}$ et d'une cale à glace de 7 à 9 tonnes de capacité, les unités de pêche ont une autonomie en mer de 7 à 9 jours (Kébé, 1982). La construction des cordiers au niveau national a été privilégiée au début du programme. Toutefois, face au non-respect des plans et caractéristiques des bateaux à la construction et le manque de performance dans l'exploitation des cordiers qui en a découlé, l'importation de prototypes a pris le dessus. $\mathrm{Au}$ total, 57 cordiers ont été mis en service par le programme, dont 35 en bois fabriqués dans les ateliers spécifiquement créés à cet effet au Sénégal et 22 métalliques importés de France et d'Espagne (Kébé, 1982).

Après quelques années d'expérimentation, le programme cordier s'est soldé par un échec considérable malgré les importantes sommes dépensées. Sa principale faiblesse tient à un manque de préparation des pêcheurs qui passent de la gestion d'une pirogue (propriété et gestion familiales, système de part prévalant pour la rémunération des matelots) à celle d'une unité semiindustrielle (propriété d'une société de pêche, salaire mensuel, primes de productivité). Les faibles performances techniques des cordiers dues au recrutement, parmi les pêcheurs artisans, de matelots inexpérimentés et donc peu performants dans une logique de pêche semi-industrielle, des pannes très fréquentes et de longues immobilisations à quai ont aussi été enregistrées. En un mot, on attendait des pêcheurs artisans un saut technologique immense dans des délais relativement courts. Toutes ces difficultés ont fini par faire sombrer cet objectif des pouvoirs publics de mettre en place une pêche semi-industrielle de démersaux côtiers. Le développement et la généralisation de la pirogue glacière (Laloë et Samba, 1990), qui relèvent d'une initiative des pêcheurs, sont allés de pair avec la dégradation des résultats économiques des cordiers. En effet, avec la péréquation sur le carburant-pêche dont le principe et les modalités sont décrits, les armateurs artisans de pirogues glacières se sont dotés de moteurs hors-bord plus puissants $(40 \mathrm{cv})$ qui ont permis l'exploitation de zones de pêche plus éloignées, dépassant même celles des cordiers, tout en allongeant la durée des marées. Les derniers bateaux cordiers opérationnels ont cessé toute activité en 1985, c'est-à-dire cinq ans avant la fin du programme prévue en 1990.

\section{Le programme sardinier}

Le premier bateau sardinier à Dakar est entré en activité en 1961. La flottille s'est progressivement développée pour atteindre un maximum de 17 bateaux au début des années 1980. Toutefois, l'année 1983 a marqué le début d'une phase de brusque régression avec une flottille de sardiniers réduite à seulement 4 unités durant les années 1990 et 2 unités à partir de 2000. En 2016, les 2 seules unités en activité ont été implantées à Ziguinchor et opèrent exclusivement dans le sud du Sénégal parce que cette région jouit de zones de pêche très côtières, d'un important potentiel halieutique de petits pélagiques côtiers et d'une demande locale soutenue aussi bien en produits frais qu'en produits transformés artisanalement.

La flottille de sardiniers a toujours été constituée de petits senneurs mesurant entre 15 et $28 \mathrm{~m}$, équipés d'une poulie hydraulique, d'un sondeur vertical et d'une radio VHF. Par définition, un senneur est un navire conçu pour la pêche à la senne, un filet destiné à capturer les espèces de poissons pélagiques par encerclement. Dans le programme sardinier, la capacité des bateaux était de 35 tonnes en moyenne avec une puissance motrice de $303 \mathrm{cv}$. Le poisson y était conservé dans de l'eau de mer refroidie par de la glace. Les sorties en mer étaient journalières et la stratégie de pêche consistait à rechercher du poisson à vue ou à l'aide d'un sondeur. La durée moyenne des sorties était d'environ 9 heures. En raison de leur faible autonomie, les sardiniers ont toujours concentré leur activité au sud de Dakar, en général sur les fonds de 10 à $50 \mathrm{~m}$ (Dème, 1988). Les pêcheurs exploitant les bateaux sardiniers étaient 
exclusivement sénégalais et la quasi-totalité de la production était destinée au marché local.

Depuis leur introduction au Sénégal, les bateaux sardiniers ont toujours présenté une situation financière déficitaire. Les recettes dégagées étaient insuffisantes pour couvrir les charges d'exploitation et d'amortissement des unités. Ce déficit était la conséquence de coûts de production élevés, de rendements faibles et d'une mauvaise gestion de la flottille. Les pannes étaient fréquentes et les immobilisations à quai très longues. Cet état de fait relevait d'un entretien défaillant et de la vétusté de la flottille constituée de bateaux d'occasion.

En tant que flottille semi-industrielle, les bateaux sardiniers n'ont jamais développé leur propre stratégie de pêche. Ils se sont adaptés aux conditions d'exploitation des unités de pêche artisanale utilisant également des sennes tournantes en termes de sorties (journalières), de temps de pêche (quelques heures), de zones de pêche (fonds peu profonds) et de détection des bancs de poisson (à vue, en général). D'ailleurs, les matelots embarqués à bord de ces sardiniers, tous issus du milieu artisanal et n'ayant bénéficié d'aucune formation supplémentaire, ont simplement reconduit leur stratégie de pêche à bord des unités artisanales de sennes tournantes. En outre, les sardiniers sont très vulnérables face à la concurrence des unités de pêche artisanale. Le poisson capturé par les sardiniers est conservé dans la glace et, au débarquement, il est de meilleure qualité que celui des sennes tournantes «artisanales». Malheureusement, les sardiniers ne bénéficient pas d'une prime de qualité et le prix de leur poisson est négativement affecté par celui de la pêche artisanale artificiellement bas. Les charges d'exploitation relativement réduites (péréquation sur le carburant, détaxation totale de l'activité de pêche), comparées à celles des sardiniers soumis à des charges de bateau industriel, expliquent ce faible niveau du prix du poisson débarqué par les unités de sennes tournantes (Dahou et al., 2000). Les capacités de débarquement des unités de senne tournante (en moyenne 3 tonnes par sortie) [Dème, 1988; Dème, 1991a] ont fini de tirer les prix vers le bas face aux possibilités d'absorption du marché de petits pélagiques côtiers. Dans la pêche artisanale prévaut le système de parts qui consiste, une fois les charges d'exploitation déduites des recettes, à répartir les revenus nets de la sortie de pêche en différentes parties égales ou non, rémunérant ainsi le travail (les pêcheurs) et le capital (pirogue, moteur et filet). Un tel système permet de partager les risques de sorties nulles entre armateurs et équipages. Par contre, dans le cas de la pêche semi-industrielle sardinière, la rémunération des matelots est régie par une convention collective. Or, cette convention définit un niveau de rémunération obligatoire quelle que soit la rentabilité financière du bateau sardinier, ce qui augmente le risque de faillite en cas de faible productivité.

\section{Les innovations fonctionnelles introduites dans la pêche artisanale}

\section{Les initiatives de remplacement de la pirogue traditionnelle}

La pirogue traditionnelle sénégalaise, creusée dans un simple tronc d'arbre et propulsée à la pagaie, a connu un certain nombre de transformations pour répondre à différents besoins de transport et de pêche. En plus de l'introduction du moteur hors-bord, les innovations majeures ont été la présence d'éperons faisant office de brise-lame et de bordés rapportés correspondant à des planches longitudinales sur les côtés permettant à la pirogue d'augmenter son volume (Chauveau, 1984). Avec une phase expérimentale entamée en 1950-1951, l'usage du moteur s'est très vite généralisé dans les pêcheries artisanales sénégalaises et, dès 1958, plus de 400 pirogues ( $20 \%$ du parc piroguier) étaient motorisées. Un large consensus s'est dégagé sur les qualités de la pirogue traditionnelle sénégalaise (Brendel, 1983; Lleres, 1986; Brendel et al., 1992). Elles sont relatives à ses grandes capacités de navigation et sa facilité de remontée et d'accostage, même sur les plages sans infrastructures. Son coût abordable pour les pêcheurs artisans dépourvus de soutien d'une institution de crédit ainsi que la disponibilité locale de la plupart des matériaux utilisés pour sa construction représentent des atouts considérables. Cependant, la pirogue traditionnelle sénégalaise comporte aussi des défauts, dont les plus apparents sont son manque de longévité, de stabilité et d'étanchéité. La nécessité d'améliorer ses qualités intrinsèques et de remédier à certains de ses défauts était devenue un impératif pour les autorités publiques sénégalaises dès les premières années de l'indépendance. Afin d'améliorer, voire de remplacer cette pirogue traditionnelle, plusieurs prototypes avaient alors été proposés par l'Etat. Des pirogues métalliques, en bois, en ferra-ciment, en fibre de verre et en résine polyester se sont succédé. Les avantages et les limites de chacun de ces prototypes sont détaillés par Brendel et al. (1992).

Suite aux différentes expériences, aucun prototype de pirogue moderne proposé n'a bénéficié de suffisamment d'atouts pour remplacer la pirogue traditionnelle. L'une des contraintes majeures de ces prototypes est que les technologies de fabrication étaient hors de portée des charpentiers sénégalais habituellement chargés de la construction et de la maintenance des pirogues. Les matériaux utilisés pour construire ces pirogues dites modernes étaient entièrement importés avec une faible valeur ajoutée locale et le coût de fabrication équivalait à presque cinq fois le prix d'une pirogue traditionnelle de même taille. Pour ces deux raisons strictement liées et en l'absence de prêts à faibles taux d'intérêt sans garanties et à rembourser sur une très longue période, il était 
quasiment impossible qu'un pêcheur artisan sénégalais, à l'assiette financière réduite, fasse l'acquisition d'un de ces prototypes.

\section{Introduction du moteur hors-bord et d'engins de pêche}

Pour la pêche artisanale, les pouvoirs publics avaient fortement concentré leurs interventions sur le segment « capture » à travers l'introduction de moteurs hors-bord et d'engins de pêche plus performants comme la senne tournante. Ces innovations technologiques s'accompagnaient d'une politique de détaxe des équipements de pêche, de péréquation sur le carburant-pêche (réduction du prix à la pompe) et d'une mise en place de structures de financement de la pêche artisanale. Il convient de noter d'ailleurs que la péréquation sur le carburant a été un facteur déterminant de la mécanisation généralisée de la pêche artisanale sénégalaise. En atténuant les coûts d'exploitation des unités de pêche, dont plus de $60 \%$ sont constitués des dépenses de carburant, cette péréquation a permis aux pêcheurs artisans l'acquisition de moteurs plus performants. Ces progrès en termes de performances motrices ont considérablement contribué à l'agrandissement de la taille des pirogues, à l'exploitation de nouvelles zones de pêche plus éloignées, dont certaines se situent dans les eaux relevant de la juridiction des autres pays ouest-africains, et à l'allongement du temps de pêche grâce à la réduction du temps de route (Dème et Dahou, 2001).

L'acquisition de pirogues de plus en plus grandes a permis l'adaptation de nouvelles techniques de pêche comme l'usage de la senne tournante. La diffusion de la senne tournante à partir de 1973, avec l'appui technique de la FAO (Laloë et Samba, 1990), a été favorisée par l'importance des ressources de petits pélagiques côtiers dans les eaux sénégalaises. C'est notamment le cas des sardinelles, de l'ethmalose, des chinchards et des maquereaux. Très abondantes, ces espèces de poissons sont fortement demandées au niveau national et régional. Par ailleurs, les limites du filet maillant encerclant en termes de capacité de captures d'une grande diversité d'espèces ont été levées avec l'adoption de la senne tournante par un plus grand nombre de pêcheurs ciblant les petits pélagiques (Fréon, 1988).

On a noté un accroissement remarquable des débarquements de petits pélagiques côtiers. Ainsi, de moins de 100000 tonnes au début des années 1980, les débarquements de ces espèces sont actuellement passés à 530175 tonnes (Fig. 1). Par contre, pour les poissons démersaux et les autres espèces (crustacés et mollusques), le volume de la production halieutique reste faible et très fluctuant. Ces progrès en matière de productivité ont induit un développement fulgurant du mareyage local (commercialisation du poisson frais) et de l'industrie de

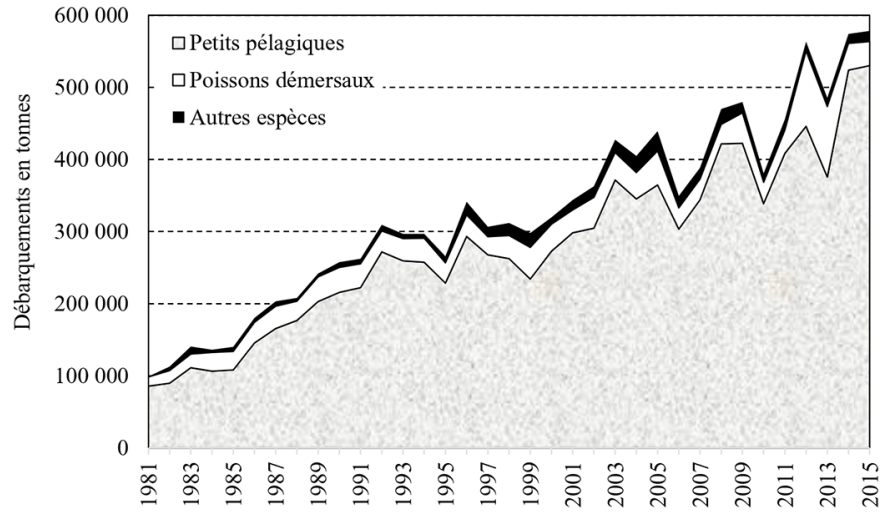

Fig. 1. Évolution des débarquements de la pêche artisanale de 1981 à 2015 (source: CRODT, 2015).

transformation artisanale du poisson tout en stimulant les exportations de produits frais et transformés dans la sousrégion ouest-africaine (Dème et Dahou, 2001; Dème et Cissé, 2016).

\section{Financement formalisé de la pêche artisanale}

En matière de financement des activités de pêche artisanale, la Caisse nationale de crédit agricole du Sénégal (CNCAS) a été mise à contribution dès 1990 . L'intervention de cette institution financière a essentiellement consisté en la gestion de lignes de crédit de divers programmes de développement de la pêche artisanale. Toutefois, les impacts de cette intervention ont été mitigés du fait de la faiblesse du portefeuille total alloué au secteur de la pêche qui, en 10 ans, n'a pas dépassé 3,2 milliards de FCFA. D'ailleurs, les conditions d'accès au crédit ont été dénoncées par les pêcheurs. En effet, outre l'exigence d'un apport personnel $(20 \%$ du montant demandé) pour l'obtention d'un crédit, le taux d'intérêt de $12,5 \%$ et les délais de paiement rigides ne tenant pas compte de la saisonnalité des activités de pêche ont souvent découragé les pêcheurs d'avoir recours à ce mode de financement.

\section{Les innovations opérationnelles impulsées par les pêcheurs artisans}

\section{Genèse des innovations impulsées par les pêcheurs}

D'importantes innovations ont vu le jour au cours des deux dernières décennies dans les pêcheries artisanales sénégalaises. Ces mutations, impulsées par les pêcheurs artisans sénégalais, constituent surtout des réponses aux baisses progressives des rendements des unités de pêche et au renchérissement des coûts de production. Ces deux phénomènes ont été liés à la surexploitation de la plupart des ressources halieutiques avec pour conséquence 
l'éloignement progressif des zones de pêche. Outre la généralisation de l'usage d'instruments technologiques d'aide à la navigation, notamment le GPS, les innovations opérées par les pêcheurs ont surtout porté sur l'introduction de nouvelles stratégies et tactiques au niveau des principales unités de pêche. Ces innovations ont été plus manifestes pour les unités de pêche à la senne tournante, la pirogue glacière, la ligne simple et le casier à seiche.

\section{Les innovations sur les unités de senne tournante}

Du fait de l'importance de la taille du filet de pêche, de celle de l'équipage et du volume des captures, l'unité type de senne tournante était généralement composée de deux pirogues. L'une des pirogues, qui mesurait environ $16 \mathrm{~m}$, était chargée de transporter le filet de pêche et une bonne partie de l'équipage. D'une longueur d'environ $18 \mathrm{~m}$, l'autre pirogue prenait en charge le transport des captures. Les deux pirogues étaient propulsées par des moteurs hors-bord d'une puissance de $40 \mathrm{cv}$. Le filet de pêche mesurait entre 300 et $400 \mathrm{~m}$ de long pour une chute de $40 \mathrm{~m}$ et capturait le poisson par encerclement.

Face au renchérissement du coût en carburant, qui compte pour plus de $70 \%$ des charges d'exploitation d'une unité de senne tournante (en moyenne 100000 FCFA par sortie journalière), les pêcheurs sont passés de deux pirogues à une seule pirogue. De taille plus grande $(22 \mathrm{~m})$, cette nouvelle pirogue avait une plus grande capacité de charge qui peut atteindre aujourd'hui 30 tonnes. Par ailleurs, d'autres innovations ont également accompagné cette décapitalisation. Parallèlement à l'agrandissement des pirogues, la longueur des filets de pêche est passée de 400 à $800 \mathrm{~m}$. Cette innovation a été particulièrement déterminante à Saint-Louis, au nord du Sénégal, où, avec l'ouverture de la brèche, les pêcheurs n'étaient plus contraints par la barre (rouleau de vagues proches du rivage) qui rendait les conditions d'embarquement, d'accostage et de débarquement très difficiles pour les grandes pirogues. En effet, en 2003, des menaces liées à une crue du fleuve Sénégal suite à des pluies diluviennes avaient poussé les autorités à créer une ouverture sur la Langue de Barbarie afin d'évacuer les eaux et de sauver la ville de Saint-Louis des risques d'inondations importantes. Ouverte à l'origine sur $4 \mathrm{~m}$, cette brèche s'étend aujourd'hui sur $7 \mathrm{~km}$ et fait désormais office d'embouchure pour les pêcheurs.

En plus de l'agrandissement du filet de senne tournante, d'autres filets ont aussi été adaptés par les pêcheurs. Une senne tournante de dimension beaucoup plus petite pour l'exploration de zones rocheuses qui abritent certaines espèces semi-pélagiques est embarquée. En outre, pour maximiser les profits de la sortie de pêche, un autre filet à petites mailles a été adopté pour cibler spécifiquement les juvéniles de sardinelles. Cette stratégie, certes illégale et destructrice, a été encouragée par la forte demande de poissons juvéniles par les industries de farine de poisson qui ont proliféré durant ces dernières années ainsi que par les commerçants originaires des pays du golfe de Guinée.

\section{Les innovations sur les unités de pirogue glacière}

L'unité de pêche à la pirogue glacière correspond à une pirogue motorisée de taille variant de 18 à $21 \mathrm{~m}$, équipée d'une caisse isotherme qui permet de conserver les captures dans la glace. Il convient de signaler que, pour ces unités de pêche, les charges d'exploitation sont relativement élevées. En outre, avec des marées pouvant atteindre 15 jours, les conditions de travail sont tellement pénibles qu'il est difficile de trouver un équipage permanent et qualifié. Par ailleurs, les pirogues glacières fréquentent le plus souvent les Zones Économiques Exclusives (ZEE) des pays voisins avec parfois des conditions d'accès draconiennes. Ces contraintes, associées à la raréfaction des ressources démersales qui constituent généralement leurs cibles, ont contribué à la baisse de la rentabilité des unités de pirogue glacière.

Pour réduire les charges d'exploitation, les pêcheurs ont opté pour une stratégie de sédentarisation en mer de la pirogue glacière. Pour cela, une deuxième pirogue est employée pour assurer le ravitaillement régulier en carburant, glace, appât, nourriture et eau. Cette pirogue ravitailleuse permet également le changement des membres d'équipage et la récupération des captures. En outre, alors que la pirogue glacière utilise habituellement la ligne et la palangre, certaines unités, qui opèrent sous licence dans les ZEE guinéennes et bissauguinéennes, embarquent désormais des filets de pêche à sole et à machoiron. Cette diversification des techniques de pêche et donc des espèces exploitées permet d'améliorer la rentabilité des unités de pêche.

\section{Les innovations sur les unités de pirogues ligne simple}

Le secteur de la pêche sénégalaise est marqué depuis quelques années par un contexte de forte surcapacité avec comme corollaire la surexploitation des principaux stocks halieutiques. Cette situation se traduit par l'éloignement des zones de pêche et la baisse des rendements. Pour s'adapter, plusieurs stratégies ont été développées par les pêcheurs travaillant à bord des pirogues à la ligne simple. Une première stratégie a consisté à recourir à une pirogue plus grande $(9 \mathrm{~m}$ au lieu de 6) et des marées de 2 à 3 jours en lieu et place de la 
sortie quotidienne qui caractérise habituellement les unités de pirogue à ligne simple. Pour conserver les captures dans la glace durant la marée, les pêcheurs ont recours à des carcasses de réfrigérateurs. Pour les sorties journalières, des caissons en polystyrène sont utilisés pour garder la fraîcheur des espèces à haute valeur marchande.

Le poulpe et diverses autres espèces démersales (mérou, dorade...), qui faisaient l'objet d'une pêche du jour (sortie quotidienne limitée à quelques heures), sont maintenant sujets à des marées de 6 jours exclusivement dans la ZEE sénégalaise à bord d'une pirogue de $14 \mathrm{~m}$ en moyenne pour 6 membres d'équipage. Le redéploiement de l'effort de pêche des pirogues de ligne normale vers la ceinture (Trichiurus lepturus), un poisson allongé pouvant dépasser $2 \mathrm{~m}$, est une autre mutation introduite par les pêcheurs artisans.

\section{Les innovations sur les unités de casiers à seiche}

La pêche artisanale céphalopodière ciblant spécifiquement la seiche s'effectue principalement à l'aide de casiers spécialement conçus à cet effet (Bakhayokho, 1981). Toutefois, les pêcheurs qui pratiquent ce type d'activité sont souvent confrontés à des pertes de casiers causées par des accrochages avec des filets maillants dérivants. Face à cette situation, nous avons pu observer sur le terrain qu'à partir de 2018 , les pêcheurs ont substitué les casiers par des sacs de riz recyclés qu'ils remplissent à moitié de sable. En outre, les pêcheurs introduisent des branches de filao dans les sacs pour attirer les seiches en période de ponte. Chaque unité de pêche dispose d'une trentaine de sacs qui sont visités quotidiennement. Cette innovation très peu coûteuse présente aussi l'avantage de capturer de seiches de plus grande taille, générant ainsi des recettes plus importantes que l'usage du casier.

\section{Les conséquences des innovations adaptatives dans la pêche artisanale}

\section{Les conséquences sur l'efficacité des systèmes d'exploitation}

Les innovations ont beaucoup contribué aux augmentations du volume des débarquements. Les évaluations des ressources pélagiques hauturières conduites annuellement par 1'ICCAT (International Commission for the Conservation of Atlantic Tunas) font état de leur pleine exploitation, voire de leur surexploitation. Le COPACE (Comité des Pêches pour l'Atlantique CentreEst) est arrivé aux mêmes conclusions concernant les petits pélagiques côtiers avec des signes de surexploitation notés pour la sardinelle (COPACE, 2017), dont les niveaux de captures sont légèrement supérieurs au rendement maximal durable $\left(\mathrm{MSY}^{3}\right)$. Ce niveau de capture ne peut donc être durablement maintenu. Une surcapacité dépassant $50 \%$ a été notée par le CRODT pour les principales démersales côtières exploitées par la pêche industrielle mais aussi la pêche artisanale (Thiam et al., 2013). Cet excès de capacité est par exemple de $55 \%$ pour le mérou blanc, de $59 \%$ pour le pageot et la sole et de $67 \%$ pour la seiche. Il convient de signaler que la surcapacité désigne la capacité d'une flotte à pêcher à des niveaux qui excèdent le niveau de capture écologiquement viable dans une zone de pêche donnée $\mathrm{du}$ fait, entre autres, d'un trop grand nombre de navires et/ou de pêcheurs mais aussi de moyens technologiques très sophistiqués. Il n'existe pas de méthode conventionnelle pour mesurer la surcapacité. Dans le cas de l'étude du CRODT, la surcapacité a été calculée à travers la méthode DEA (data envelopment analysis) ou méthode d'analyse par enveloppement des données en français. Cette méthode permet d'estimer le taux d'efficience des unités de pêche qui, s'il est supérieur à $100 \%$, dénote une surcapacité.

$\mathrm{Au} \mathrm{vu}$ des résultats issus de l'estimation de la surcapacité des pêcheries démersales côtières sénégalaises, l'augmentation continue des captures peut sembler paradoxale. En fait, les innovations technologiques ont permis de cibler de nouvelles espèces (ceinture, merlu, rouget...) présentant un certain intérêt commercial lié à une demande extérieure soutenue face à la baisse des espèces traditionnellement pêchées (machoiron, otolithe, pageot, mérou, pagre, capitaine, seiche...) et de redéployer davantage l'effort de pêche dans les ZEE des autres pays de la sous-région. Il faut cependant souligner que cette stratégie de redéploiement des pêcheurs sénégalais dans les pays voisins a été facilitée par la politique de péréquation sur le carburant instituée depuis plusieurs décennies.

\section{Les innovations comme moteur d'adaptabilité et de durabilité}

Plusieurs travaux ont démontré la capacité des pêcheurs artisans sénégalais à imaginer de nouvelles tactiques et stratégies de pêche pour s'adapter aux variabilités spatiotemporelles de l'abondance et de la

3 Le maximum sustainable yield (MSY) appelé en français rendement maximal durable (RMD) ou encore rendement maximal soutenable (RMS) est la plus grande quantité de biomasse que l'on peut extraire en moyenne et à long terme d'un stock halieutique dans les conditions environnementales existantes sans affecter le processus de reproduction et donc de renouvellement de l'espèce concernée. 
demande des ressources (Pech et al., 2001; Brochier et al., 2018; Le Fur, 2019). Afin de s'accommoder au mieux des modifications du capital naturel, ces innovations adaptatives constantes du capital humain et technologique dénommé «capital créé par les hommes » (man-made capital) seraient assimilables au principe de «durabilité faible» qui fonde l'exploitation des ressources naturelles, notamment halieutiques (Solow, 1993; Faucheux et Noël, 1995; Godard, 1994). Pionnier de la notion de «durabilité faible», Solow (1993) considère qu'on n'a pas besoin de conserver telle ou telle ressource, encore moins de conserver tous les éléments naturels dans un état inaltéré, mais plutôt de préserver de façon indéfinie la capacité productive des sociétés humaines, étant entendu que le capital de la société ne se limite pas aux équipements productifs (usines, machines, bâtiments) mais comprend tous les actifs qui contribuent à engendrer du bien-être dans le futur: savoir et compétences (capital humain), mais aussi actifs naturels. Cette théorie se base sur le caractère de substituabilité entre capital naturel et capital humain, contrairement à la «durabilité forte» qui suppose une complémentarité de ces capitaux. Cette dernière reconnaît la nécessité de définir un noyau minimal de capital naturel critique à entretenir et à transmettre en tant que tel aux générations futures.

Il convient de noter que les approches relatives aux notions de durabilité faible et de durabilité forte ont été développées dans le cadre de modèles macroéconomiques très abstraits et ne tenant pas compte du principe d'innovation. Par contre, dans le cas particulier de notre article, ces notions s'appliquent à un secteur d'activité spécifique et donc à une échelle méso-économique dans laquelle est intégrée la question de l'innovation. Ce changement d'échelle et de paradigme semble donc apporter des réponses concrètes quant à la validité de ces modèles de durabilité. Cette prétention mérite toutefois d'être considérée avec prudence car la transposition d'un raisonnement de l'échelle macroéconomique à l'échelle méso-économique pourrait se confronter à certaines contraintes fonctionnelles qui n'ont pas été étudiées dans notre travail.

\section{Conclusion}

En dépit de quelques progrès considérables, les politiques publiques de développement de la pêche artisanale sénégalaise ont connu des succès relativement mitigés. Dans le cadre de deux importants projets, les tentatives de substitution des sennes tournantes et de la pêche à la ligne par respectivement des bateaux sardiniers et des cordiers ont échoué. En effet, contrairement à ces unités dites modernes, les unités traditionnelles de pêche artisanale disposent d'importants atouts qui garantissent leur viabilité et leur résilience. D'abord, leur organisation et leur mode de fonctionnement permettent le partage des risques économiques entre armateurs et équipages. Ensuite, l'importance des pertes est souvent atténuée par leurs coûts d'exploitation relativement faibles du fait de la détaxe des équipements de pêche et la péréquation sur le carburant. Par ailleurs, les unités traditionnelles disposent de plusieurs facilités de renouvellement des équipements de pêche à travers des taux d'intérêt bonifiés, la présence de mutuelles d'épargne et de crédit et surtout le préfinancement des mareyeurs et des transformateurs de produits halieutiques. Tous ces facteurs font que leur rentabilité a toujours dépassé celles des sardiniers et des cordiers de nature semiindustrielle en permettant aux pêcheurs de tirer pleinement parti de leur connaissance de l'environnement naturel. Ces bateaux sont restés cloisonnés dans un système de pêche industrielle (taxation, impôt, convention collective, etc.) très rigide qui ne leur permet pas de s'adapter facilement aux conditions changeantes d'exploitation des ressources halieutiques côtières, contrairement aux unités de pêche artisanale. Ainsi, même si l'intervention publique est indispensable pour orienter et réguler le secteur de la pêche, il serait important de maintenir la capacité de gouvernance interne de la pêche artisanale en veillant à ce que les incitations proposées par les autorités s'appuient autant que possible, sans lui nuire, sur cette adaptabilité. En effet, pour améliorer l'efficience des activités de pêche, un certain nombre d'innovations ont été introduites par les pêcheurs euxmêmes. Les principales réussites de la pêche artisanale en termes de modernisation résident cependant dans la motorisation des pirogues et l'introduction de la senne tournante destinée à l'exploitation des petits pélagiques côtiers.

L'aide généralisée sous forme de détaxe des intrants et la péréquation sur le carburant ont permis une extension progressive de l'effort de pêche au-delà de l'optimum économique de la plupart des ressources exploitées. À ce stade de la gestion des ressources halieutiques sénégalaises, rien ne justifie donc plus le maintien de ce soutien financier public généralisé. Il conviendrait d'instaurer une discrimination positive en faveur des unités de pêche artisanale, ciblant les petits pélagiques côtiers, qui sont tournées, en grande partie, vers la satisfaction de la demande nationale en protéines animales. Une telle stratégie permettrait de s'approcher de l'objectif de sécurité alimentaire que l'État a toujours assigné à la pêche artisanale sénégalaise et d'atténuer l'effort de pêche sur les ressources démersales côtières fortement surexploitées. L'orientation d'une partie des débarquements de petits pélagiques côtiers vers l'industrie de farine de poisson constitue une menace sérieuse pour cette politique nationale et pour la durabilité des pêcheries. Selon les autorités, cette industrie reste encore 
concentrée et à des niveaux contenus sur le traitement des déchets des usines de transformation, mais elle incite fortement à l'exploitation des juvéniles.

D'une manière générale, il convient de retenir qu'au Sénégal, la pression de pêche ne cesse de s'accroître et aggrave encore la situation des ressources halieutiques. Ainsi, les nombreuses innovations adaptatives mises en œuvre dans les pêcheries artisanales s'inscrivent de fait dans une dynamique de durabilité environnementale faible. Originellement conceptualisée dans un cadre macroéconomique, l'application de cette théorie de substituabilité entre capital naturel et capital humain à l'échelle méso-économique mérite toutefois d'être appréhendée avec une certaine prudence dans la mesure où toutes les propriétés fonctionnelles de ce raisonnement n'ont pas été étudiées dans notre travail.

\section{Références}

Bakhayokho M., 1981. Historique des pêcheries de céphalopodes (seiches, poulpes, calmars) des côtes sénégalaises, Pêche maritime, 1224, 634-640.

Bradley D., Merrifield M., Miller K.M., Lomonico S., Wilson J.R., Gleason M.G., 2019. Opportunities to improve fisheries management through innovative technology and advanced data systems, Fish and Fisheries, 20, 3, 564-583, https://doi.org/10.1111/faf.12361.

Brendel R., 1983. Perspectives d'amélioration de la pirogue sénégalaise. Rapport technique, Dakar, Direction de l'océanographie et des pêches maritimes.

Brendel R., Kebe M., Deme M., 1992. Modernisation de la pêche artisanale. Bilan des tentatives de remplacement et/ ou d'amélioration de la pirogue traditionnelle sénégalaise, Document scientifique du CRODT, 138.

Brochier T., Auger P., Thiao D., Bah A., Ly S., Nguyen-Huu T., Brehmer P., 2018. Can overexploited fisheries recover by self-organization? Reallocation of fishing effort as an emergent form of governance, Marine Policy, 95, 46-56.

Charneau D., 1988. L'économie du thon au Sénégal: intégration nationale et internationalisation de la filière, Document scientifique du CRODT, 109.

Chauveau J.-P., 1984. La pêche piroguière sénégalaise: les leçons de l'histoire, Revue Mer, numéro spécial, 10-15.

Chauveau J.-P., 1989. Histoire de la pêche industrielle au Sénégal et politiques d'industrialisation: des origines aux premières années de l'indépendance, Cahier des Sciences Humaines, 25, 1-2, 237-258.

COPACE (Comité des pêches pour l'Atlantique Centre-Est), 2017. Rapport sur l'état des ressources dans la zone COPACE, Synthèse, Rome, FAO.

CRODT (Centre de recherches océanographiques de DakarThiaroye), 2015. Statistiques de la pêche maritime sénégalaise en 2015: pêche artisanale, pêche thonière et pêche chalutière, Archive scientifique du CRODT, 226.

Dahou K., Dème M., Dioum A., 2000. Impact socioéconomique et environnemental des dispositifs d'appui aux pêches sénégalaises, Document technique, PNUD, ENDA tiers monde.

Dème M., 1988. Étude économique et financière de la pêche sardinière sénégalaise, Document scientifique du CRODT, 107.

Dème M., 1991a. Les effets du soutien financier de l'État à la pêche artisanale: le cas du Sénégal, in Durand J.-R., Lemoalle J., Weber J. (Eds), La recherche face à la pêche artisanale. Tome II, Paris, ORSTOM, 845-849.

Dème M., 1991b. Les politiques d'investissement et d'intervention de l'État Sénégalais dans le secteur des pêches: problématique générale et actions de recherches, Document scientifique du CRODT, 128.

Dème M., Cissé I., 2016. Organisation de la chaîne d'approvisionnement du marché ouest-africain des petits pélagiques, impacts des entreprises étrangères sur ces marchés et sur les communautés, Rapport final, Greenpeace.

Dème M., Levenez J.-J., 1991. L'exploitation des petits pélagiques au Sénégal: réponse de la recherche à la crise sardinière, in Durand J.-R., Lemoalle J., Weber J. (Eds), La recherche face à la pêche artisanale. Tome II, Paris, ORSTOM, 657-669.

Dème M., Dahou K., 2001. Subventions et développement durable des pêcheries au Sénégal. Communication à l'atelier sur L'impact des politiques nationales et européennes de pêche sur la sécurité alimentaire des populations des pays en développement, 12-13 juin, Dakar, CRODT/CEMARE.

Domingo J., 1982. Deux expériences de développement de la pêche maritime au Sénégal, Les Cahiers d'Outre-Mer, 35, 35-62, www.persee.fr/doc/caoum_0373-5834_1982_ num_35_137_3008.

Faucheux $\overline{\text { S}}$., Noël J.-F., 1995. Économie des ressources naturelles et de l'environnement, Paris, Armand Colin.

Fréon P., 1988. Réponses et adaptations des stocks de clupléidés d'Afrique de l'Ouest à la variabilité du milieu et de l'exploitation: analyse et réflexion à partir de l'exemple du Sénégal, Paris, ORSTOM.

Godard O., 1994. Le développement durable: paysage intellectuel, Natures Sciences Sociétés, 2, 4, 309-322, https://doi.org/10.1051/nss/19940204309.

Hall S.J., Hilborn R., Andrew N.L., Allison E.H., 2013. Innovations in capture fisheries are an imperative for nutrition security in the developing world, Proceedings of the National Academy of Sciences of the United States, 110, 21, 8393-8398, https://doi.org/10.1073/pnas.1208067110.

Kébé M., 1982. La pêche cordière au Sénégal, Document scientifique du CRODT, 81.

Laloë F., Samba A., 1990. La pêche artisanale au Sénégal: ressource et stratégies de pêche, Paris, ORSTOM.

Le Fur J., 2019. Computer exploration of factors involved in the viability of a fishery sector: the case of the small-scale fresh fish supply in Senegal at the end of the 20th century, in Barrière O., Behnassi M., David G. (Eds), Coviability of social and ecological systems: reconnecting mankind to the biosphere in an era of global change, Cham, Springer. 
Lleres B., 1986. La pêche piroguière maritime au Sénégal. Son évolution, son introduction dans l'économie de marché. Thèse de doctorat en géographie, Bordeaux, Université de Bordeaux-III.

OCDE (Organisation de coopération et de développement économiques), 2005 [1 $1^{\mathrm{re}}$ éd. 1997]. Manuel d'Oslo: principes directeurs pour le recueil et l'interprétation des données sur l'innovation, Paris, OCDE.

Pech N., Samba A., Drapeau L., Sabatier R., Laloë F., 2001. Fitting a model of flexible multifleet-multispecies fisheries to Senegalese artisanal fishery data, Aquatic Living Resource, 14, 81-98, https://doi.org/10.1016/S0990-7440 (01)01108-1.

Solow R., 1993. An almost practical step toward sustainability, Resources Policy, 19, 3, 162-172, https:/doi.org/10.1016/ 0301-4207(93)90001-4.

Thiam N., Fall M., Thiaw M., 2013. Étude sur l'état des pêcheries côtières, des stocks clés et de leurs habitats, Rapport technique, Dakar, PRAO-SN/DPM.

Citation de l'article : Dème M., Thiao D. Politiques de pêche et innovations adaptatives des pêcheries artisanales sénégalaises. Nat. Sci. Soc. 29, 2, 174-184. 